\title{
Performance Analysis of Cooperative Hybrid Cognitive Radio Network with Various Diversity Techniques
}

\author{
C. S. Preetham, M. S. G. Prasad, D. S. S. L. Saranya, Charan Teja Somepalli, \\ D. Bhargava Satya Sai Krishna, V. Rohit \\ Department of Electrical and Communication Engineering, Koneru Lakshmaiah University
}

\begin{tabular}{l}
\hline Article Info \\
\hline Article history: \\
Received Apr 8,2016 \\
Revised May 18, 2016 \\
Accepted Jun 4, 2016 \\
\hline Keyword: \\
Cognitive radio \\
Equal gain combining \\
Signal to noise ratio combining \\
Fading and path loss \\
Maximal ratio combining \\
Optimum weight adaptation \\
\hline
\end{tabular}

\begin{abstract}
The extensive growth in wireless communications leads to spectrum scarcity. Since the spectrum is limited spectrum usage is clogged. The best possible solution is usage of cognitive radio. A cognitive radio network with sender, receiver and intermediate devices as relays is analyzed. The channel is modelled with noise considerations, path loss and variance. The system is defined with one primary sender and one primary receiver, in between them five secondary users and two active users. The signals from all these paths are estimated and analyzed to draw the best signal with good signal to noise ratio (SNR). To improve the channel efficiency and quality, we have considered various diversity techniques for which the fading problem of channel can be eliminated. In view of this, we concentrated on improving the system performance with various diversity techniques and optimum weight adaptation concept.
\end{abstract}

Copyright $(2016$ Institute of Advanced Engineering and Science. All rights reserved.

\section{Corresponding Author:}

C. S. Preetham,

Department of Electrical and Communication Engineering,

Koneru Lakshmaiah University,

Green Fields, Vaddeswaram, Guntur, Andhra Pradesh, India 522502.

Email: cspreetham@kluniversity.in

\section{INTRODUCTION}

The considerable problem of development in wireless networks is spectrum scarcity. This is because poor utilization of spectrum. So we have to look at alternative solution that can use spectrum intelligently. Cognitive Radio networks [1] will be the best solution as far as possible [2] to increase the spectrum resources in wireless applications by understanding Dynamic Spectrum Access (DSA) [3]. Various techniques are discovered to get the access of the spectrum by both primary users (PU) and secondary users (SU) simultaneously. They are underlay, overlay and interweave. Among them, Underlay is best and flexible. Moreover the Secondary user's transmit power have limited interference. To avoid this limitation we go for the AF amplify and forward technique. In spite of the advantages in underlay method, advances are limited. Based on usage of the PU spectrum by secondary users (SU), overlay, underlay, interweave are discovered.

In underlay approach Transmissions occur at the same time in the both users [4] and Primary user's receiver sets the interference threshold to Secondary User [5]. The range of communications is limited because of this interference threshold. To improve this relays are to be placed in between Primary user and Secondary user. Within the interference range the secondary user can share the spectrum of primary user. Underlay gives the maximum benefits to secondary users only.

The secondary user acts like a relay in overlay method to transfer the data from PU transmitter to Primary receiver. So we can notice some improvement in SNR. To transmit its own data some part of secondary user power is used and remaining is for relaying the data of primary user. No restriction to interference. Both the users transmits simultaneously by giving more priority to primary users (PU) [6] with no restriction on interference. 
Secondary users find the spectrum holes in primary user bandwidth in interweave technique [7]. Instead of competing for accessing the spectrum, primary user guides the secondary user in accessing spectrum using spectrum mobility, spectrum sensing and spectrum management [8].Sensing stage is for searching the spectrum holes [9]. The best channel and the best relay are decided in spectrum management stage by the receiver. Once the spectrum hole is in usage and if it is again wanted by primary user then the decision is to be taken by secondary user which is controlled by spectrum mobility.

Implementation of relays, not only increases the performance but also the primary user energy is saved [10]. Switching of Hybrid underlay/overlay is studied by S.senthuran et.al which improves secondary user's outturn [11]. Junni Zau et.al proposed multiple secondary users which are having a relay for transmission of its own data [12]. In order to improve the outturn dynamic change of mode by secondary user is conferred by Authors Hojin song et.al [13]. For transmission of secondary user mode is decided by the PU activity. Until the detection of transmission by primary user, SU will be in overlay mode.

Diversity technique is, at receiver if there are more number of incoming signals with same rush of data, they are combined into a single improved signal [14]. There are combining techniques like Equal gain combining (EGC), Maximum ratio combining (MRC), Signal to noise ratio combining (SNRC), etc. By using the optimum weight adaptation for MRC will improve performance of system when compared to conventional diversity techniques [14].

Our entire paper is considered as below. System model is proposed in section 2. Section 3 provides the algorithm for hybrid relaying. The diversity techniques are discussed in section4. Results are analyzed in section 5. Conclusions are drawn in final section 6.

\section{SYSTEM MODEL}

We propose a CR system which is having Primary transmitter $\left(\mathrm{PT}_{\mathrm{X}}\right)$ and Primary receiver $\left(\mathrm{PR}_{\mathrm{X}}\right)$. In addition, there are many active and inactive secondary users. Figure 1 shows the proposed system model.

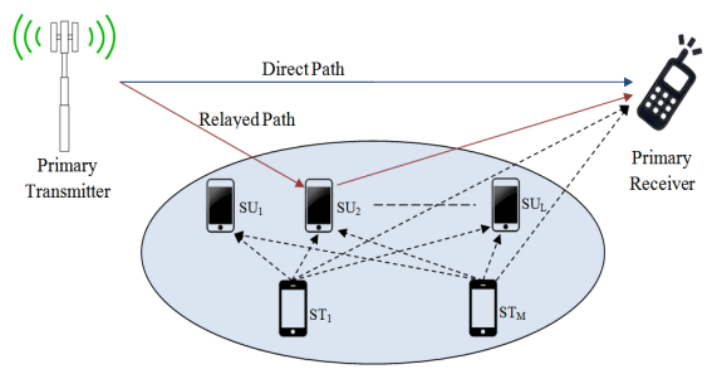

Figure 1. System Model

It consists of ' $L$ ' and ' $M$ ' inactive and active secondary users. One best relay must be selected among ' $\mathrm{L}$ ' inactive users. Among the secondary users only interference is generated by active users and relaying is done by inactive users. Active users are represented as $\mathrm{ST}_{\mathrm{i}}$ where $\mathrm{i}=1,2 . \mathrm{M}$ and inactive users are represented as SU's. SU's transmits the data to destinations throughout the time. Relay path is selected whenever the target rate of relay path is more than the target rate of direct path. Now the transmission takes place in 2 fragments using the best relay. Among the $\mathrm{k}$ channels and $\mathrm{L}$ inactive users one channel and the best relay are to be selected with the condition that interference to the PU should be minimum value. The entire powers of the secondary users are used to transmit the data of PU. In partial relay selection the source transmits the data is sent to all inactive users [15]. Among all those one best relay is selected by the Primary receiver. Let $\mathrm{P}_{\mathrm{PT}}$ and $\mathrm{P}_{\mathrm{ST}}$ are transmit powers of Primary transmitter and Primary receiver respectively.

\section{ALGORITHM OF PROPOSED MODEL}

The proposed model of hybrid relay suggests the scheme for choice of most effective relay [16]. The PU transmitter power, interference limit, distance between PU and SU, and distance between users are taken into the consideration of the algorithm. First of all the formulations, the target rate of direct path is calculated between the users. 
Let $\alpha_{P T_{x}-P R_{x}}, \alpha_{P T_{x}-S U_{j}}, \alpha_{S T_{i}-P R_{x}}, \alpha_{S U_{j}-P R_{x}}$ and $\alpha_{S T_{i}-S U_{j}}$ are the gains of channels of links $\mathrm{PT}_{\mathrm{x}} \rightarrow \mathrm{PR}_{\mathrm{x}}, \mathrm{ST}_{\mathrm{i}} \rightarrow \mathrm{PR}_{\mathrm{x}}, \mathrm{PT}_{\mathrm{x}} \rightarrow \mathrm{SU}_{\mathrm{j}}, \mathrm{SU}_{\mathrm{j}} \rightarrow \mathrm{PR}_{\mathrm{x}}$, and $\mathrm{ST}_{\mathrm{i}} \rightarrow \mathrm{SU}_{\mathrm{j}}$. Let the gap dependent path loss issue is n. Let $\mathrm{PRx}$ is received power which is associated with the $\mathrm{P}_{\mathrm{PT}}$ transmitted power by $\mathrm{PT}_{\mathrm{x}}$ as

$$
P_{P R}=\frac{\alpha_{P T_{\chi}-P R_{X} P} P T}{\left(d_{\left.P T_{X}-P R_{X}\right)^{n}}\right.}
$$

$\mathrm{d}_{\text {PTx-PRx }}$ signifies the gap between the primary $\mathrm{Tx}$ and primary $\mathrm{Rx}$. The power strength of interference is $\mathrm{P}^{\prime}$ ' at Primary receiver by active secondary user STi is formulated as

$$
P_{i}^{\prime}=\frac{\alpha_{S T_{i}-P R_{X} P} S T_{i}}{\left(d_{S T_{i}-P R X}\right)^{n}}
$$

The distance between active secondary user and primary receiver is $\mathrm{d}_{\mathrm{PTx} \text {-PRx }}$. So the SNIR $S N I R_{P T_{x}-P R_{x}}$ on the primary receiver of $P T_{x}-P R_{x}$ is outlined as

$$
\operatorname{SNIR}_{P T_{x}-P R_{x}}=\frac{P P R}{\sum_{i=1}^{M} P_{i}^{\prime}+\sigma_{p}^{2}}
$$

The AWGN variance of primary $\mathrm{T}_{\mathrm{x}}$ to $\mathrm{R}_{\mathrm{x}}$ is $\sigma \_\mathrm{p}^{\wedge} 2$. The attainable $R_{\text {target }}$ rate in bits/s/Hz of link $\mathrm{PT}_{\mathrm{x}}-\mathrm{PR}_{\mathrm{x}}$ is outlined

$$
R_{\text {target }}=\log _{2}\left(1+S N I R_{P T_{x}-P R_{x}}\right)
$$

Out of all the inactive users, $\mathrm{R}_{\mathrm{ej}}$ will be the most effective relay to send the data of primary user. $\mathrm{P}_{\mathrm{PT}}$ is the transmitted power of $\mathrm{PT}_{\mathrm{x}}$, then the received power at secondary inactive user $\mathrm{SUj}$ is represented by

$$
P_{S U_{j}}=\frac{\alpha_{P T_{x}-S U_{j} P_{P_{T}}}}{\left(d_{P T_{X}-S U_{j}}\right)^{n}}
$$

$d_{P T_{x}-S U_{j}}$ Is the distance between the $\mathrm{PT}_{\mathrm{x}}$ and also the inactive secondary user. The interference is created at SU' due to active users. That power strength $p_{i j}^{\prime}$ of interference on $S U_{j}$ is given by

$$
p_{i j}^{\prime}=\frac{\alpha_{S T_{i}-S U_{j}} P_{S T_{i}}}{\left(d_{S T_{i}-S U_{j}}\right)^{n}}
$$

The interference by user ' $i$ ' to user ' $\mathrm{j}$ ' is $p_{i j}^{\prime}$ and the distance between the active and idle is SU $d_{S T_{i}-S U_{j}}$. The primary Tx transmits the information to relays on different channels. The speed of information arrival at idle $\mathrm{SU}$ is

$$
R_{P, S U_{j}}=\frac{1}{2} \log \left(1+\frac{P_{S U_{j}}}{\sigma_{j}^{2}+\sum_{i=1}^{M} p_{i j}^{\prime}}\right)
$$

Where $\sigma_{j}^{2}$ is variance of AWGN on PU $\mathrm{T}_{\mathrm{x}}$ to idle $\mathrm{SU}$. For each relay $\mathrm{SU}_{\mathrm{j}}$ that is associated with each subcarrier $\mathrm{k}$ estimates the ability needed to urge a similar rate in supply to relay and then secondary path of destination via relay.

$$
p_{j, k}^{\text {rate }}=\frac{\left(2^{\left({ }^{\left(2 R_{P, S U_{j}}\right)}-1\right)\left(\sigma_{k}^{2}+\sum_{i=1}^{M} p_{i j}^{\prime}\right)\left(d_{S U_{j}-P R_{\chi}}\right)^{n}}\right.}{\alpha_{S U_{j}-P R_{X}}}
$$

Where $d_{S U_{j}-P R_{x}}$, is the distance between idle SU and the PU's receiver and $\sigma_{k}^{2}$ is that variance of AWGN on idle SU's to PU's receiver. For every ' $\mathrm{j}$ ' "relay and ' $\mathrm{k}$ 'th' channel notice utmost power that may is allotted to every relay.

$$
p_{j, k}^{\max }=\frac{I_{t h}}{\Omega_{j, k}}
$$


$\Omega_{j, k}$ is reason of channel interference and $I_{t h}$ is the threshold limit of interference, the factor of interference $\Omega_{j, k}$ is

$$
\Omega_{j, k}=\alpha T_{s} \int_{d_{k}{ }^{-B} / 2}^{d_{k^{+}} B / 2}\left(\sin \pi f T_{s} /_{\pi f T_{s}}\right)^{2} d f
$$

Where $\alpha$ is channel gain, sampling time $\mathrm{T}_{\mathrm{s}}$, distance between the subcarrier $\mathrm{k}$ and the PU channel be $\mathrm{d}_{\mathrm{k}}$, bandwidth reserved by the PU channel [17] be B. The power allocated to each relay $\mathrm{SU}_{\mathrm{j}}$ over the channel $\mathrm{k}$ is given by

$$
\operatorname{power}_{j, k}=\min \left(p_{j, k}^{\max }, p_{j, k}^{\text {rate }}\right)
$$

The power of signal at PU destination is

$$
\operatorname{power}_{j, k}^{R e}=\frac{\alpha_{S U_{j}-P R_{x} P^{\text {Power }}}{ }_{j, k}}{\left(d_{S U_{j}-P R_{x}}\right)^{n}}
$$

The pair $(\mathrm{j}, \mathrm{k})$ is the optimal relay and channel to relay since has max value in (14).

$$
\begin{aligned}
& \left(j^{o p t}, k^{o p t}\right)=\operatorname{argmax}\left(\text { power }{ }_{j, k}^{R e}\right) \\
& \left(j^{o p t}, k^{o p t}\right)=\operatorname{argmax}\left(\frac{\left|h_{i}\right|^{2}\left|g_{i}\right|^{2} P_{r} P_{S}}{\left|h_{i}\right|^{2} P_{S} \sigma_{S}^{2}+\left|g_{i}\right|^{2} P_{r}^{\sigma^{2}}}\right)
\end{aligned}
$$

The signal rate at PU receiver from the optimal relay and relay-channel pair is given by

$$
R_{P R_{x}}=\frac{1}{2} \log \left(1+\frac{\text { power }_{j}^{R e p t}, k^{o p t}}{\sigma_{k}^{2}+\sum_{i=1}^{M} p_{i}^{\prime}}\right)
$$

If $R_{P R_{x}}>R_{\text {target }}$ then direct path transmission is neglected and relayed path is considered. The best signal among these two signals is having good power and maximum SNR. The system performance is increased by suppressing the poor signal and using the best signal among them.

\section{DIVERSITY COMBINING TECHNIQUES}

Previously many researchers used either direct or relayed signal at receiver. But now we want to implement the diversity techniques like EGC, MRC, and SNRC at the receiver side to combine both the direct and relay signals, so that to improve the channel capacity [18]. In EGC, all the $\mathrm{R}_{\mathrm{x}}$ signals are just added. Among all the diversity methods EGC is the simplest way. When we discuss about the performance levels of combining techniques EGC will definitely have low performance.

$$
\mathrm{y}_{\mathrm{d}}(\mathrm{n})=\sum_{i=1}^{k} y_{i, d}(n)
$$

$y_{i, d}(n)$ Represents the different signals received at the receiver. As we are taking the direct path and relay path there will be only two signals. Then the equation will be

$$
y_{d}(n)=y_{s, d}(n)+y_{r, d}(n)
$$

Where $\mathrm{y}_{\mathrm{s}, \mathrm{d}}(\mathrm{n})$ is $\mathrm{R}_{\mathrm{x}}$ signal from sender and $\mathrm{y}_{\mathrm{r}, \mathrm{d}}(\mathrm{n})$ is the signal from the relay. If we weight the coefficients in a brilliant way then the better performance can be achieved. Often, the parameter used to estimate the quality of a link is SNR. The expression for this is

$$
\mathrm{y}_{\mathrm{d}}(\mathrm{n})=\sum_{i=1}^{k} S N R y_{i, d}(n)
$$

As we are taking the direct path and relay path there will be only two signals. Then the equation will be

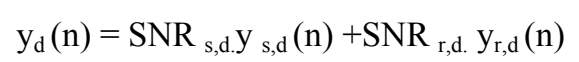


where $\mathrm{SNR}_{\mathrm{s}, \mathrm{d}}$ denotes the $\mathrm{S} / \mathrm{N}$ ratio of the direct link, $\mathrm{SNR}_{\mathrm{r}, \mathrm{d}}$ the relay channel, $\mathrm{y}_{\mathrm{s}, \mathrm{d}}(\mathrm{n})$ signifies the $\mathrm{R}_{\mathrm{x}}$ signal from the $T_{x}$ and $y_{r, d}(n)$ is the from the relay. Above all the methods are having less channel capacity when compared to MRC.

MRC is known to be of high performance at receiver as the weighs of input signal are taken from their channel statistics. The best possible performance is achieved by using MRC. In this the each input signal is multiplied with its respective channel gain.

$$
\mathrm{Y}_{\mathrm{d}}(\mathrm{n})=\sum_{i=1}^{k} \mathrm{~h}_{\mathrm{i}, \mathrm{d}}{ }^{*}(\mathrm{n}) \cdot \mathrm{y}_{\mathrm{i}, \mathrm{d}}(\mathrm{n})
$$

Using direct and relay signal, the equation becomes

$$
\mathrm{Y}_{\mathrm{d}}(\mathrm{n})=\mathrm{h}_{\mathrm{s}, \mathrm{d}}{ }^{*}(\mathrm{n}) \cdot \mathrm{y}_{\mathrm{s}, \mathrm{d}}(\mathrm{n})+\mathrm{h}_{\mathrm{r}, \mathrm{d}}{ }^{*}(\mathrm{n}) \cdot \mathrm{y}_{\mathrm{r}, \mathrm{d}}(\mathrm{n})
$$

Where $\mathrm{h}_{\mathrm{s}, \mathrm{d}}{ }^{*}(\mathrm{n})$ is the conjugate of direct signal gain, $\mathrm{h}_{\mathrm{r}, \mathrm{d}}{ }^{*}(\mathrm{n})$ is the conjugate of relay signal gain, $\mathrm{y}_{\mathrm{s}, \mathrm{d}}$ (n) is the $R_{x}$ signal from the $T_{x}$ and $y_{r, d}(n)$ is from relay. But according to the practical considerations the conjugate of channel gain is $0.097^{\wedge}(0.5)$. So the result will not be accurate as the channel gain is very less. For achieving improved capacity we used weight adaptation techniques like Keiser, Gaussian, and Binomial [19].

Binomial weights will create no side lobes. The rows of pascal's triangle are chosen as binomial coefficients. The coefficients are arranged such that

$$
\mathrm{a}_{\mathrm{nr}} \equiv \frac{\mathrm{n} !}{\mathrm{r} !(\mathrm{n}-\mathrm{r}) !} \equiv\left(\begin{array}{c}
\mathrm{n} \\
\mathrm{r}
\end{array}\right)
$$

Where $\left(\begin{array}{l}\mathrm{n} \\ \mathrm{r}\end{array}\right)$ is binomial coefficient. As the first and third coefficients are equals to 1 then the MRC value equals to EGC. That's why we are neglecting the binomial coefficients.

Gaussian is important in most areas. The points close to the center are taken as weighted coefficients. The Gaussian is expressed as

$$
\Omega(\mathrm{x})=\mathrm{e}^{\left(-\frac{(\mathrm{x}-\mu)^{2}}{2 \sigma^{2}}\right)}
$$

$\mu$ represents center location and $\boldsymbol{\sigma}$ represents closest value. The area under curve is highly concentrated and so less weights at tails. In general the Gaussian is expressed as

$$
\mathrm{w}_{\mathrm{n}}(\mathrm{k}+1)=\mathrm{e}^{-\frac{1}{2}\left(\alpha \frac{\mathrm{k}-\frac{\mathrm{N}}{2}}{\frac{\mathrm{N}}{2}}\right)^{2}}
$$

Finally the channel capacities of different combining techniques by varying $I_{\text {th }}$ values, graphs are plotted.

Kaiser Bessel function is having the best values when compared to other techniques. Weights are determined by

$$
\mathrm{W}_{\mathrm{n}}(\mathrm{k})=\frac{\mathrm{I}_{0}\left[\pi \alpha \sqrt{1-\left(\frac{\mathrm{K}}{\mathrm{N}}\right)^{2}}\right]}{\mathrm{I}_{0}[\pi \alpha]}
$$

\begin{tabular}{|c|c|c|c|c|c|c|c|c|}
\hline \multirow{2}{*}{$\begin{array}{l}\text { Array weight } \\
\text { Function }\end{array}$} & \multicolumn{7}{|c|}{ Normalized weights } & \multirow{2}{*}{$\begin{array}{l}\text { Weighted } \\
\text { Sum }\end{array}$} \\
\hline & $\mathrm{W}_{1}$ & $\mathrm{~W}_{2}$ & $\mathrm{~W}_{3}$ & $\mathrm{~W}_{4}$ & $\mathrm{~W}_{5}$ & $\mathrm{~W}_{6}$ & $\mathrm{~W}_{7}$ & \\
\hline Binomial & 1 & 1 & 1 & 1 & 2 & 1 & 1 & 2.546 \\
\hline Gaussian & 0.8825 & 0.9382 & 0.9773 & 0.9975 & 0.9975 & 0.9773 & 0.9382 & 4.010 \\
\hline $\begin{array}{l}\text { Kaiser-Bessel with } \\
\qquad \alpha=1\end{array}$ & 1 & 0.9974 & 0.9897 & 0.9768 & 0.9583 & 0.9340 & 0.9035 & 5.251 \\
\hline $\begin{array}{l}\text { Kaiser-Bessel with } \\
\qquad \alpha=3\end{array}$ & 1 & 0.9975 & 0.9894 & 0.9768 & 0.9583 & 0.9340 & 0.9035 & 7.359 \\
\hline
\end{tabular}

Where $\mathrm{k}=0,1,2,3 \ldots$ and $\alpha>1 . \alpha$ is an arbitrary, non-negative real number that determines the shape of the window. In the frequency domain, it determines the trade-off between main-lobe width and side lobe level, which is a central decision in window design. When compared to all 4 weighting techniques Kaiser Bessel function gives best performance, as shown in Table 1 .

Table 1. Adaptive weights of different windows with $\mathrm{N}=7$

Performance Analysis of Cooperative Hybrid Cognitive Radio Network with Various .... (C. S. Preetham) 


\section{SIMULATION RESULTS}

The PRx and $\mathrm{PT}_{\mathrm{x}}$ are placed at $(500,40)$ and $(4,40)$, as shown in Figure 2. The 5 idle Secondary User's and the 2 active Secondary Users are placed at $(200,20),(400,20),(100,40),(200,40),(250,40)$, $(300,40),(400,40)$ serially. Let us consider that, power of $\mathrm{ST}_{\mathrm{i}}$ is $P_{S T_{1}}=P_{S T_{2}}=10 \mathrm{~dB}$ and power of Primary User's transmitter is $P_{P T}=10 \mathrm{~dB}$. Path loss issue is taken as n, link gain $(\alpha)$ and variance $\left(\sigma^{\wedge} 2\right)$ are taken as $\left(0.097 / d^{2}\right)^{1 / 2}$ and $10^{-13}$. The Relayed channels are of $1 \mathrm{MHz}$ and for PU channel is $2 \mathrm{MHz}$.

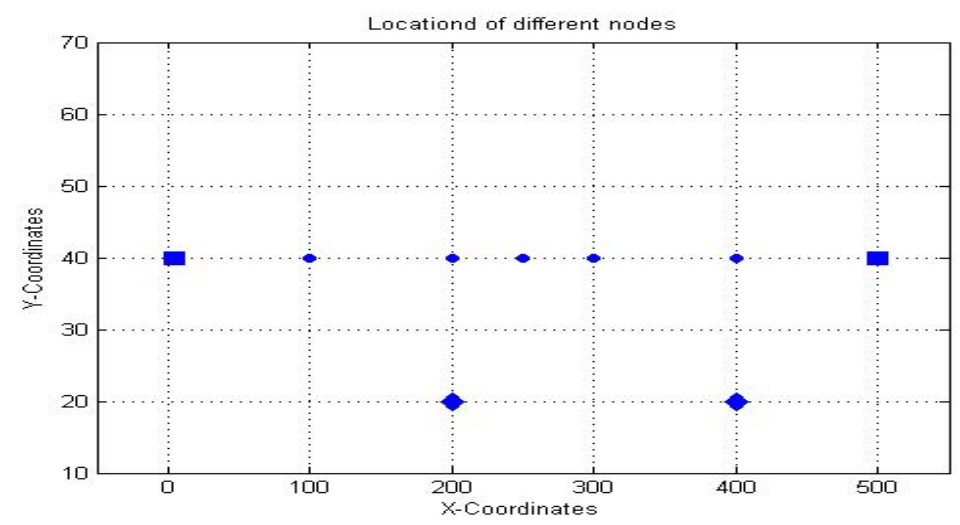

Figure 2. Location of different nodes

\subsection{Analysis of Partial Relay Selection}

Figure 3 relates the capability of the most efficient relay to the variable interference threshold. Up to $3 \mathrm{~mW}$ the overlay technique has the high capacity and direct path has low capacity. But if we increase the threshold the interference increases in overlay. In spite of increasing the Interference threshold the benefit of the hybrid relay transmission over the overlay and underlay transmission ways is represented in Figure 2.

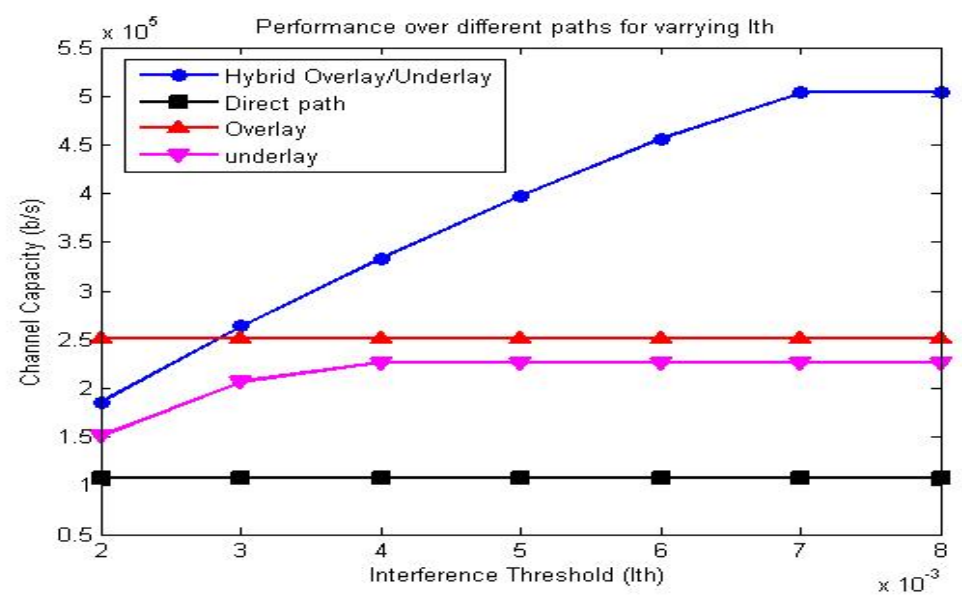

Figure 3. channel capacity vs interference threshold for different hybrid relay paths

From Figure 4 it is seen that the hybrid relay choice criterion is in a position to deliver higher capability than interference as constraint, however it is less capability when compared to power as constraint. The benefit in the planned model is that it can produce sensible capability and causes less interference to the other active PU's. 


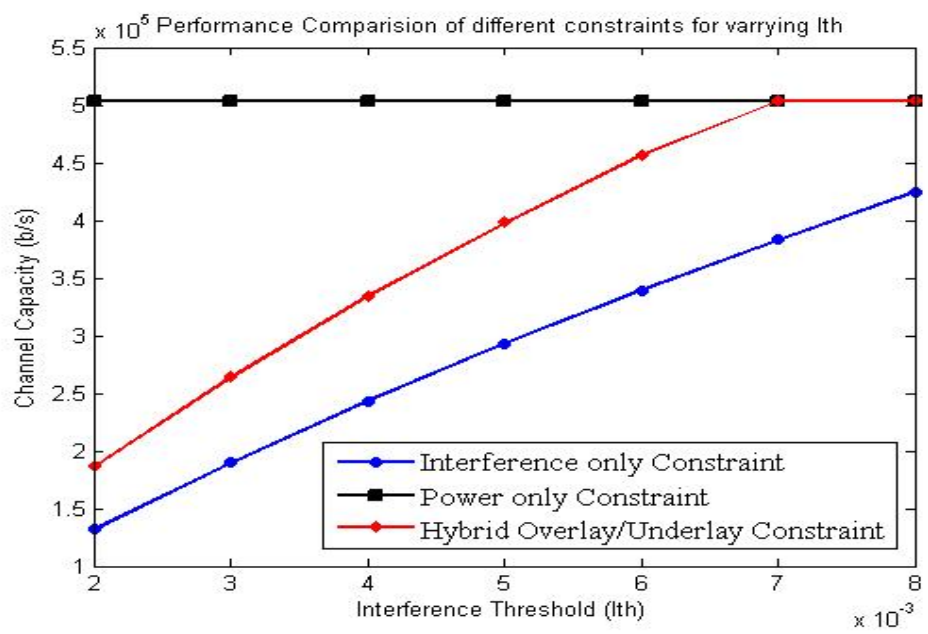

Figure 4. Channel capacity vs interference threshold for different constraints

This is for capability of different paths in variance with PU transmitter power as shown in Figure 5. The direct path is linear when compared to the other paths. By comparing the opportunistic and partial methods, up to $10 \mathrm{~dB}$ partial has the better performance. And the opportunistic has the better performance after 10dB. Because the opportunistic relay selection has two channels and hence we have two SNRs. So in this method the two SNRs are added and the curve capacity increases with increase in power.

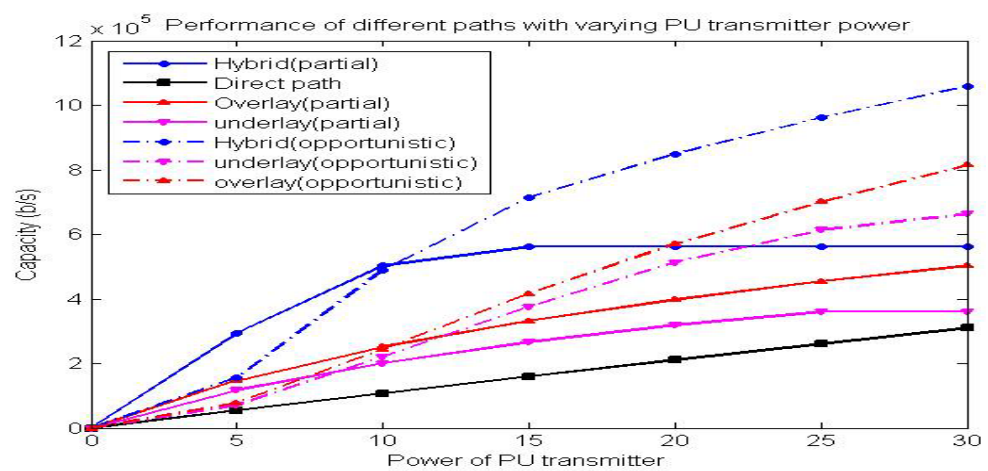

Figure 5. Channel capacity vs transmitter power for different hybrid relays

\subsection{Implementation of Diversity Techniques in Relayed Networks}

Figure 6 shows the implementation of MRC using windowing techniques like Gaussian, Keiser, Binomial techniques. Performance of MRC using binomial weights is similar to the performance of MRC using EGC. So by comparing Gaussian and Keiser, Keiser gives the optimum results for getting channel capacity of MRC. The channel capacity is gradually increased till the $\mathrm{I}_{\text {th }}$ values equals to $7 \mathrm{~mW}$. After $7 \mathrm{~mW}$ the channel capacity becomes a constant value. 


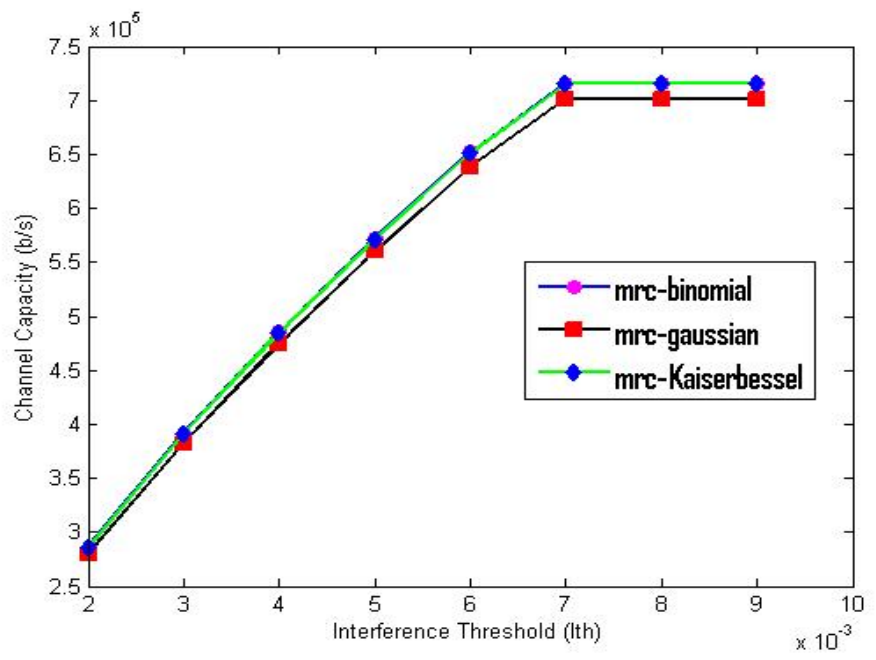

Figure 6. channel capacity vs interference threshold for MRC using optimum weights

Figure 7 shows the performance of hybrid overlay/underlay technique with different diversity techniques such as EGC, SNRC and MRC. Analysis of the combining techniques is done against the Hybrid overlay/underlay without combining techniques. From the graph it is observed that MRC using Kaiser Bessel weights is having better channel capacity than all the other combining techniques. The performance of Hybrid CR technique without use of combining techniques is low. The capacity is significantly improved when combining techniques are implemented.

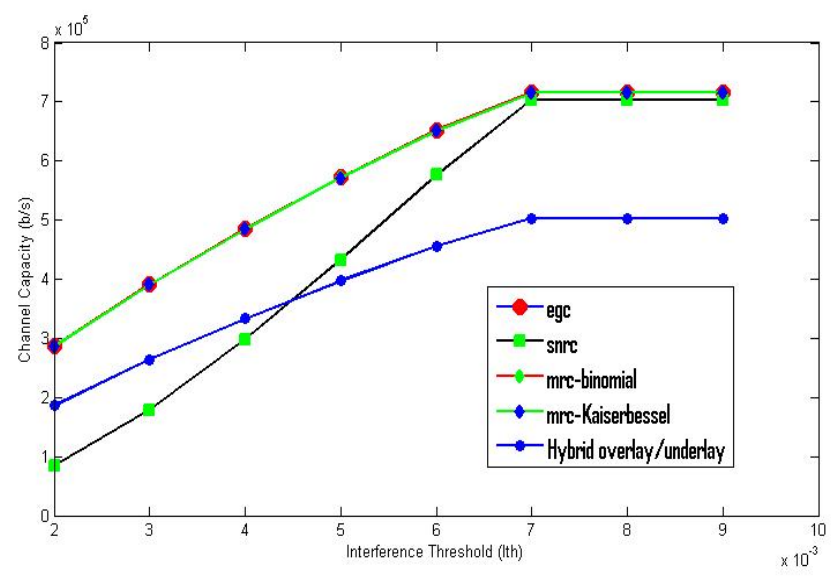

Figure 7. channel capacity vs interference threshold for diversity techniques

\section{CONCLUSION}

Cognitive radio is the most effective solution for usage of white spaces in spectrum by secondary user. Till now relaying is done either by overlay and underlay method. These methods are incompatible with diversity techniques. We in this paper implemented the hybrid relaying in cognitive radio networks. The proposed hybrid relay network removes the switching problem faced by the previous hybrid relay networks. This hybrid relay network has advantages of both the relaying methods. The hybrid relaying has given an opportunity to introduce diversity techniques in Cognitive radio relaying. By introducing these techniques system performance is increased. In this paper, we implemented adaptive diversity techniques for the first time to combine the direct path and relayed path signals at receiver. In these diversity techniques weights are calculated using adaptive weighting algorithms such as binomial, Gaussian and Kaiser-Bessel. These measures have given significant improvement in the system performance. The work in this manuscript clearly shows that hybrid overlay/underlay relaying technique by the use MRC with keiserbessel weight 
adaptation technique at the PU receiver can give significantly better performance than the traditional overlay, underlay and hybrid overlay/underlay without combining methods.

\section{REFERENCES}

[1] K. C. C. a. R. Prasad, "Cognitive Radio Networks,” England, John Wiley \& Sons Ltd, 2009.

[2] J. Mitola and G. Q. Maguire, "Cognitive radio: making software radios more personal," IEEE Personal Communications, vol. 6, pp. 13-18, 1999.

[3] S. Force, "Spectrum policy task force report," Federal Communications Commission ET Docket 02, vol. 135, 2002.

[4] C. S. Preetham and M. S. G. Prasad, "Relay, power and subchannel allocations for underlay non LOS OFDM-based cognitive networks under interference temperature," pp. 205-209, 2015.

[5] M. Xia and S. Aissa, "Underlay cooperative af relaying in cellular networks: performance and challenges," IEEE Communications Magazine, vol. 51, pp. 170-176, 2013.

[6] L. Lu, et al., "Ten years of research in spectrum sensing and sharing in cognitive radio," EURASIP Journal on Wireless Communications and Networking, vol. 2012, pp. 28, 2012.

[7] X. Tan, et al., "Achievable Transmission Rate of the Secondary User in Cognitive Radio Networks with Hybrid Spectrum Access Strategy,” IEEE Communications Letters, vol. 17, pp. 2088-2091, 2013.

[8] I. F. Akyildiz, et al., "NeXt generation/dynamic spectrum access/cognitive radio wireless networks: A survey," Computer Networks, vol. 50, pp. 2127-2159, 2006.

[9] T. H. Chuang, et al., "Alleviating Interference through Cognitive Radio for LTE-Advanced Network," International Journal of Electrical and Computer Engineering vol. 5, pp. 539-547, 2015.

[10] W. Su, et al., "Active Cooperation Between Primary Users and Cognitive Radio Users in Heterogeneous Ad-Hoc Networks," IEEE Transactions on Signal Processing, vol. 60, pp. 1796-1805, 2012.

[11] S. Senthuran, et al., "Throughput Analysis of Opportunistic Access Strategies in Hybrid Underlay\&\#x2014; Overlay Cognitive Radio Networks," IEEE Transactions on Wireless Communications, vol. 11, pp. 2024-2035, 2012.

[12] J. Zou, et al., "Optimal Power Allocation for Hybrid Overlay/Underlay Spectrum Sharing in Multiband Cognitive Radio Networks," IEEE Transactions on Vehicular Technology, vol. 62, pp. 1827-1837, 2013.

[13] H. Song, et al., "On the Optimal Switching Probability for a Hybrid Cognitive Radio System," IEEE Transactions on Wireless Communications, vol. 12, pp. 1594-1605, 2013.

[14] M. S. G. Prasad, et al., "Analysis of different direction of arrival (DOA) estimation techniques using smart antenna in wireless communications," pp. 639, 2009.

[15] J. Jia, et al., "Cooperative Relay for Cognitive Radio Networks,” pp. 2304-2312, 2009.

[16] M. Usman and I. Koo, "Access Strategy for Hybrid Underlay-Overlay Cognitive Radios With Energy Harvesting," IEEE Sensors Journal, vol. 14, pp. 3164-3173, 2014.

[17] Y. Wang, et al., "A Hybrid Underlay/Overlay Transmission Mode for Cognitive Radio Networks with Statistical Quality-of-Service Provisioning," IEEE Transactions on Wireless Communications, vol. 13, pp. 1482-1498, 2014.

[18] Anusha M., et al., "Transmission protocols in Cognitive Radio Mesh Networks," International Journal of Electrical and Computer Engineering, vol. 5, pp. 1446-1451, 2015.

[19] A. Bletsas, et al., "Cooperative Communications with Outage-Optimal Opportunistic Relaying," IEEE Transactions on Wireless Communications, vol. 6, pp. 3450-3460, 2007. 